\title{
Somnolence and Sedation Were Transient Adverse Events for Most Patients Receiving Clobazam Therapy: Post Hoc Analysis of Trial OV-1012 Data
}

\author{
James Benjamin Renfroe ${ }^{\mathrm{a}, \mathrm{c}}$, Jouko Isojarvi ${ }^{\mathrm{b}}$, Deborah Lee ${ }^{\mathrm{b}}$
}

\begin{abstract}
Background: Somnolence-related adverse events (AEs) are common with benzodiazepine treatment. Clobazam is a uniquely structured 1,5-benzodiazepine indicated in the US for the adjunctive treatment of seizures associated with Lennox-Gastaut syndrome (LGS) in patients $\geq 2$ years old. This post hoc analysis of the phase III OV-1012 trial evaluated the incidence, as well as time to onset and resolution, of somnolence and/or sedation AEs following treatment with clobazam for patients with LGS.
\end{abstract}

Methods: OV-1012 was a double-blind, placebo-controlled study that compared three oral dosages of clobazam with placebo as adjunctive therapy in patients $2-60$ years of age with LGS. Following a 4-week baseline phase, patients who had $\geq 2$ drop seizures per week were randomized to placebo or clobazam $0.25,0.5$, or 1.0 $\mathrm{mg} / \mathrm{kg}$ /day (low-dosage, medium-dosage, and high-dosage, respectively). Treatment included a 3 -week titration phase, followed by a 12-week maintenance phase. Incidence, time to onset, duration, and time to resolution of somnolence/sedation AEs were analyzed for patients treated with placebo, or low-, medium-, or high-dosage clobazam.

Results: The incidence of somnolence and/or sedation was greater for patients treated with any dosage of clobazam (26\%) than placebo $(15 \%)$, and the incidence increased with greater dosages of clobazam (low-dosage, 17\%; medium-dosage, 27\%; and high-dosage, 32\%). For most patients, onset of these events was within the first 3 weeks of treatment, corresponding to the titration phase. The majority of these events resolved (placebo, 73\%; low-dosage, 82\%; medium-dosage, $63 \%$; and high-dosage, $83 \%$ ). The median duration of these somnolence/sedation AEs was 1 month for all clobazam treatment groups.

Conclusions: Somnolence and sedation were relatively common

Manuscript accepted for publication August 11, 2015

${ }^{a}$ Child Neurology Center of Northwest Florida, Gulf Breeze, FL, USA

bLundbeck LLC, Deerfield, IL, USA

${ }^{\mathrm{c}}$ Corresponding Author: James Ben Renfroe, Child Neurology Center of Northwest Florida, Clinical Research Group, ECV Research, 400 Gulf Breeze Parkway, Suite 300, Gulf Breeze, FL 32561, USA.

Email: ben.renfroe@cneurology.com

doi: http://dx.doi.org/10.14740/jnr334w
AEs observed during clobazam treatment for LGS in OV-1012 and were dosage-related. Most of these events were transient and resolved within a few weeks.

Keywords: Clobazam; Lennox-Gastaut syndrome; Post hoc analyses; Somnolence; Sedation; Safety

\section{Introduction}

Lennox-Gastaut syndrome (LGS) is a severe epilepsy of childhood characterized by slow spike-and-wave electroencephalogram (EEG), several seizure types (including tonic and atonic), and significant developmental delay and behavioral problems $[1,2]$. Patients with LGS often require lifelong polytherapy with antiepileptic drugs (AEDs) to manage multiple seizure types [1,3]. Classic 1,4-benzodiazepines are used to manage several seizure types, but somnolence-type adverse events (AEs) are common with this class of drug $[4,5]$.

Clobazam is a 1,5-benzodiazepine indicated in the United States for adjunctive treatment of seizures associated with LGS in patients 2 years of age or older [6]. The efficacy and safety of clobazam was evaluated in phase III trial OV-1012 [7]. Clobazam $0.25,0.5$, and $1.0 \mathrm{mg} / \mathrm{kg}$ /day (low-, medium-, and high-dosages, respectively) were evaluated as adjunctive therapy in patients with LGS. The incidence of AEs with $\geq$ $10 \%$ difference between placebo and clobazam at any dosage was somnolence, pyrexia, lethargy, drooling, and constipation. No new safety issues versus historical reports were observed.

Data from earlier trials show that somnolence and sedation AEs are commonly associated with clobazam treatment [8]. This post hoc analysis of OV-1012 data evaluated the incidence, as well as time to onset and resolution, of somnolence/ sedation AEs.

\section{Materials and Methods}

\section{Study background}

Data for this post hoc analysis of somnolence and sedation 

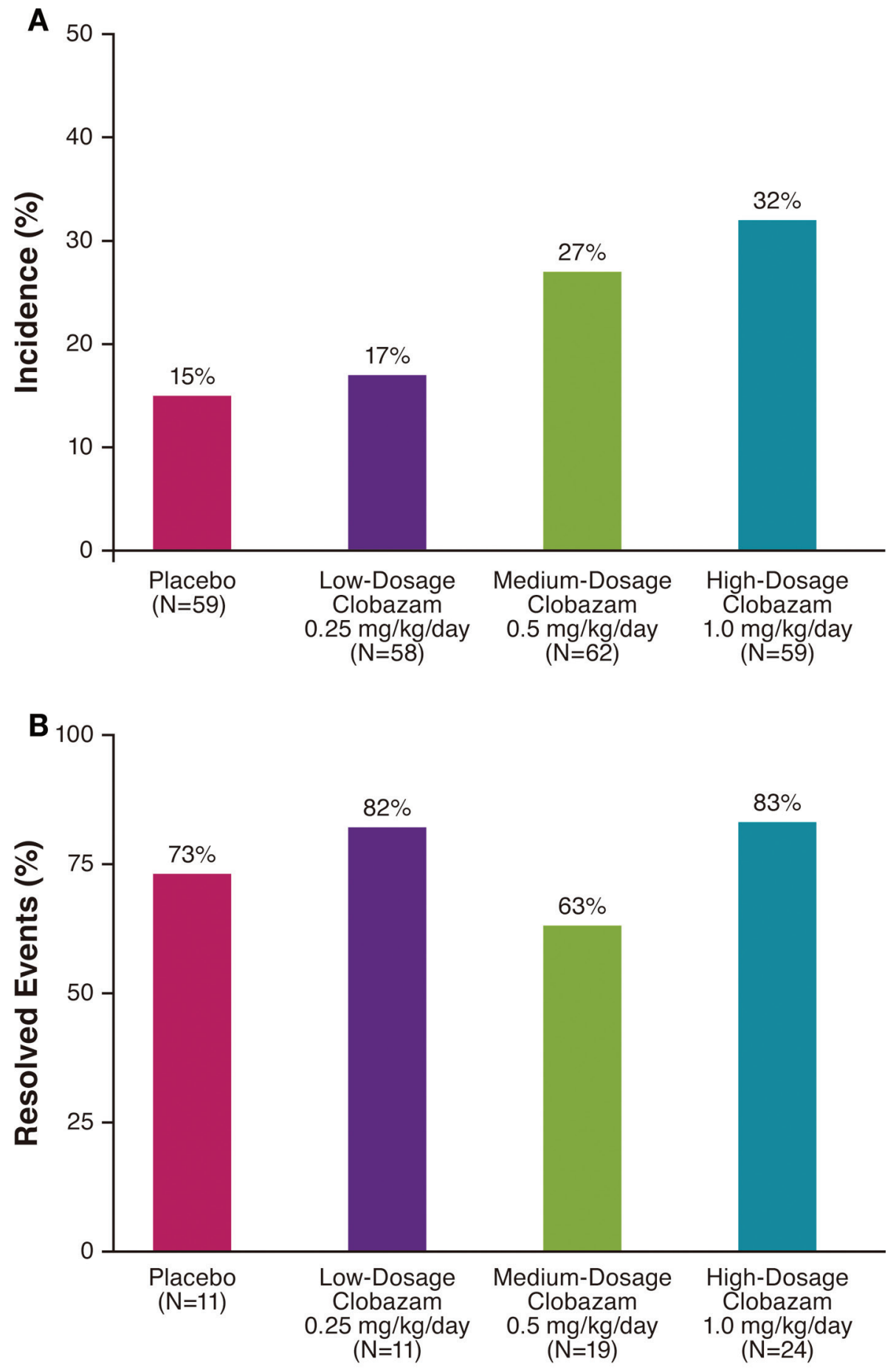

Figure 1. Incidence (A) and resolution (B) of somnolence/sedation adverse events by treatment group.

rates were obtained from trial OV-1012 (NCT00518713 [7]). OV-1012 was a phase III, multicenter, randomized, doubleblind, placebo-controlled, parallel-group study conducted in patients 2 - 60 years of age with a diagnosis of LGS. Eligible patients had LGS onset at $<11$ years of age and were receiving stable dosages of 1 - 3 AEDs (except benzodiazepines) for $\geq 30$ days. The study included a 4 -week baseline period, a 3-week titration period, and a 12-week maintenance period. Patients who experienced $\geq 2$ drop seizures during the baseline period were stratified by weight $(12.5 \mathrm{~kg}$ to $\leq 30 \mathrm{~kg}$ and $>30$ $\mathrm{kg}$ ) and then randomized to one of three clobazam treatment groups (low-dosage: target of $0.25 \mathrm{mg} / \mathrm{kg} /$ day (maximum 10 $\mathrm{mg}$ /day)), medium-dosage: target of $0.5 \mathrm{mg} / \mathrm{kg} /$ day (maximum $20 \mathrm{mg} /$ day), and high-dosage: target of $1.0 \mathrm{mg} / \mathrm{kg} /$ day (maxi- 
mum $40 \mathrm{mg} /$ day)) or placebo.

\section{Post hoc analysis}

Safety data from all patients in OV-1012 who received $\geq 1$ dose of study drug or placebo (safety population) were analyzed. The AE database was searched using the Medical Dictionary of Regulatory Activities (MedDRA) preferred terms of "somnolence" and "sedation". Analyses included incidence of somnolence/ sedation AEs (occurring after the first dose of study drug and within 30 days after the last dose of study drug), and $\mathrm{AE}$ resolution. Kaplan-Meier estimates of survival function were used to analyze time to onset of first somnolence/sedation $\mathrm{AE}$, and time to AE resolution.

\section{Results}

\section{Patient demographics and clinical characteristics}

A total of 301 patients were screened in trial OV-1012, and 238 were randomized and comprised the safety population (placebo: 59 patients; clobazam low-dosage: 58; medium-dosage: 62; and high-dosage: 59). One hundred seventy-seven patients completed the study.

A total of 55 patients experienced somnolence/sedation AEs (placebo: nine patients, clobazam low-dosage: 10; medium-dosage: 17; and high-dosage: 19). The mean age for these patients was 13.5 years (range: 2 - 49), and mean baseline weekly drop-seizure rate was 78.0 seizures (range: 2 - 856). The most commonly used concomitant AEDs (incidence $\geq$ $30 \%$ ) were multiple forms of valproic acid/valproate (45.5\%), lamotrigine $(40.0 \%)$, and levetiracetam $(38.2 \%)$. Patient demographic data and the pattern of concomitant AED distribution in this subpopulation were similar to the overall trial population [7].

\section{Somnolence and sedation AEs}

A total of 54 somnolence/sedation AEs were observed in $26 \%$ (46/179) of clobazam-treated patients in the safety population. The overall incidence of somnolence and/or sedation was greater with clobazam treatment than placebo (15\%), and the incidence of these events was proportional to clobazam dosage, with the lowest clobazam dosage yielding a similar percentage to placebo (Fig. 1A). For most patients, first onset of somnolence/sedation AEs occurred during the 3-week titration period (Fig. 2A).

The majority of somnolence/sedation AEs resolved by the end of the study in all treatment groups (Fig. 1B). AE resolution was not proportional to clobazam dosage, and the greatest rates of resolution were observed in patients receiving lowor high-dosage clobazam ( $82 \%$ and $83 \%$, respectively). In all clobazam-treated patients, most of the somnolence/sedation AEs resolved within about 60 days, and the median duration of these events was 1 month (Fig. 2B).

\section{Discussion}

Somnolence/sedation AEs are commonly associated with classic 1,4-benzodiazepines [4,5], as well as the 1,5-benzodiazepine clobazam [8]. To explore the incidence of somnolence and sedation following treatment with adjunctive clobazam in patients with LGS, AE data from trial OV-1012 [7] were evaluated in this post hoc analysis.

The incidence of somnolence/sedation AEs in the low-dosage clobazam group was similar to that of the placebo group, and was lesser than those in the medium- and high-dosage clobazam treatment groups. Further, the incidences of somnolence and sedation in the greater-dosage clobazam treatment groups were similar to the incidence of sedation (about 26\%) calculated from a summary of 70 double-blind studies of clobazam (the incidence of sedation for diazepam was about 46\% [9]). As expected, the first onset of somnolence and/or sedation occurred during the 3-week titration period. By the end of the study, the majority of somnolence/sedation AEs had resolved, most notably in the low- and high-dosage clobazam treatment groups ( $>$ $80 \%$ ). In all clobazam-treated patients, most of the somnolence/ sedation AEs resolved within about 60 days.

The overall rate of somnolence/sedation AEs observed in this post hoc analysis for all patients treated with clobazam was $26 \%$. Results from two studies (a randomized controlled trial and an open-label study) showed that approximately $67 \%$ of patients with epilepsy treated with clonazepam, a 1,4-benzodiazepine indicated for the treatment of LGS, complained of drowsiness in the first week of treatment with clonazepam [10]. Patient drowsiness in the two clonazepam studies improved after the first week despite a gradual increase in dosage, and $<10 \%$ of patients experienced drowsiness after 3 weeks of treatment [10]. In OV-1012, 32\% of patients who received high-dosage clobazam experienced somnolence and/or sedation. Although these data suggest that clobazam treatment might be associated with a lesser incidence of somnolence/ sedation AEs than clonazepam treatment, this comparison is limited and must be interpreted with caution, given that the trials vary in design, type of epilepsy studied, identification of patients affected, and patient population.

\section{Conclusions}

This post hoc analysis of trial OV-1012 data found that somnolence/sedation AEs were relatively common and occurred more frequently with greater dosages of clobazam. The majority of these AEs occurred during the 3-week titration period, and most events resolved within a few weeks of treatment.

\section{Acknowledgement}

The authors would like to thank Rebecca Drummond, PhD, and Guangbin Peng, PhD, of Lundbeck LLC (Deerfield, IL) for their assistance with data generation and analyses. Manuscript preparation, including editing and formatting the manuscript, incorporating author comments, preparing tables and 

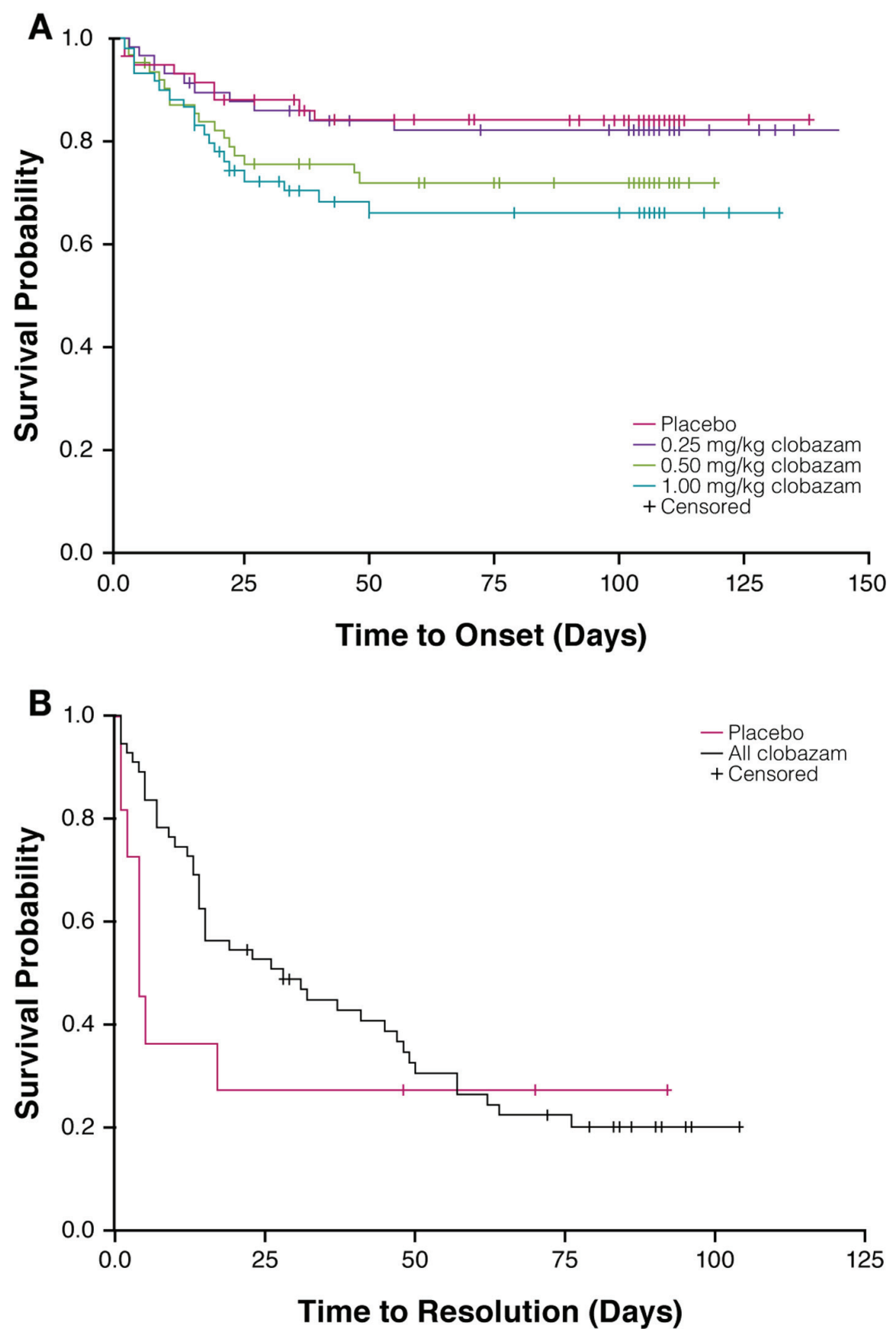

Figure 2. Time to onset of first somnolence/sedation adverse event by treatment group (A) and time to resolution of somnolence/ sedation adverse events in clobazam-treated patients (B).

figures, and coordinating submission requirements, was provided by Apurva Dave, $\mathrm{PhD}$, of Prescott Medical Communications Group (Chicago, IL).

\section{Disclosures and Conflicts of Interest}

This study was funded by Lundbeck LLC (Deerfield, IL). Drs. Isojarvi and Lee are Lundbeck employees. Dr. Renfroe is a
Lundbeck research investigator.

\section{References}

1. Arzimanoglou A, French J, Blume WT, Cross JH, Ernst JP, Feucht M, Genton P, et al. Lennox-Gastaut syndrome: a consensus approach on diagnosis, assessment, management, and trial methodology. Lancet Neurol. 
2009;8(1):82-93.

2. Glauser TA. Following catastrophic epilepsy patients from childhood to adulthood. Epilepsia. 2004;45(Suppl 5):23-26.

3. Montouris GD. Rational approach to treatment options for Lennox-Gastaut syndrome. Epilepsia. 2011;52(Suppl 5):10-20.

4. Kennedy GM, Lhatoo SD. CNS adverse events associated with antiepileptic drugs. CNS Drugs. 2008;22(9):739760.

5. Riss J, Cloyd J, Gates J, Collins S. Benzodiazepines in epilepsy: pharmacology and pharmacokinetics. Acta Neurol Scand. 2008;118(2):69-86.

6. Onfi (clobazam) prescribing information. Deerfield, IL: Lundbeck LLC; 2013.
7. Ng YT, Conry JA, Drummond R, Stolle J, Weinberg MA. Randomized, phase III study results of clobazam in Lennox-Gastaut syndrome. Neurology. 2011;77(15):14731481.

8. Giarratano M, Standley K, Benbadis SR. Clobazam for treatment of epilepsy. Expert Opin Pharmacother. 2012;13(2):227-233.

9. Koeppen D. A review of clobazam studies in epilepsy. In: Hindmarch I, Stonier PD, Trimble MR, editors. Clobazam, human psychopharmacology and clinical applications. Royal Society of Medicine. 1985:1207-1215.

10. Nanda RN, Johnson RH, Keogh HJ, Lambie DG, Melville ID. Treatment of epilepsy with clonazepam and its effect on other anticonvulsants. J Neurol Neurosurg Psychiatry. 1977;40(6):538-543. 